\title{
The dark cube: Dark and light character profiles
}

Danilo Garcia, Patricia Rosenberg

Background: Research addressing distinctions and similarities between people's malevolent character traits (i.e., the Dark Triad: Machiavellianism, narcissism, and psychopathy) has detected inconsistent linear associations to temperament traits. Additionally, these dark traits seem to have a common core expressed as uncooperativeness. Hence, some researchers suggest that the dark traits are best represented as one global construct (i.e., the unification argument) rather than as ternary construct (i.e., the uniqueness argument). We put forward the dark cube (cf. Cloninger's character cube) comprising eight dark profiles that can be used to compare individuals who differ in one dark character trait while holding the other two constant. Our aim was to investigate in which circumstances individuals who are high in each one of the dark character traits differ in Cloninger's "light" character traits: self-directedness, cooperativeness, and self-transcendence. We also investigated if people's dark character profiles were associated to their light character profiles.

Method: A total of 997 participants recruited from Amazon's Mechanical Turk (MTurk) responded to the Short Dark Triad and the Short Character Inventory. Participants were allocated to eight different dark profiles and eight light profiles based on their scores in each of the traits and any possible combination of high and low scores. We used three-way interaction regression analyses and t-tests to investigate differences in light character traits between individuals with different dark profiles. As a second step, we compared the individuals' dark profile with her/his character profile using an exact cell-wise analysis conducted in the ROPstat software ( http://www.ropstat.com ).

Results: Individuals who expressed high levels of Machiavellianism and those who expressed high levels of psychopathy also expressed low self-directedness and low cooperativeness. Individuals with high levels of narcissism, in contrast, scored high in selfdirectedness. Moreover, individuals with a profile low in the dark traits were more likely to end up with a profile high in cooperativeness. The opposite was true for those individuals with a profile high in the dark traits. The rest of the cross-comparisons revealed some of the characteristics of human personality as a non-linear complex dynamic system. 
Conclusions: Our study suggests that individuals who are high in Machiavellianism and psychopathy share a unified non-agentic and uncooperative character (i.e., irresponsible, low in self-control, unempathetic, unhelpful, untolerant), while individuals high in narcissism have a more unique character configuration expressed as high agency and, when the other dark traits are high, highly spiritual but uncooperative. In other words, based on differences in their associations to the light side of character, the Dark Triad seems to be a dyad rather than a triad. 
2

3

4

5

6

7

8

9

The Dark Cube: Dark and Light Character Profiles

Danilo Garcia ${ }^{1,2,3,4 *}$, Patricia Rosenberg ${ }^{3 *}$

${ }^{1}$ Blekinge Center of Competence, Blekinge County Council, Karlskrona, Sweden

13

${ }^{2}$ Department of Psychology, University of Gothenburg, Gothenburg, Sweden

14

${ }^{3}$ Network for Empowerment and Well-Being, Lyckeby, Sweden

15

${ }^{4}$ Institute of Neuroscience and Physiology, Sahlgrenska Academy, Gothenburg, Sweden

16

${ }^{2}$ Department of Psychology, Lund University, Lund, Sweden

17

18

19

20

21

22

23 
Background: Research addressing distinctions and similarities between people's malevolent character traits (i.e., the Dark Triad: Machiavellianism, narcissism, and psychopathy) has detected inconsistent linear associations to temperament traits. Additionally, these dark traits seem to have a common core expressed as uncooperativeness. Hence, some researchers suggest that the dark traits are best represented as one global construct (i.e., the unification argument) rather than as ternary construct (i.e., the uniqueness argument). We put forward the dark cube (cf. Cloninger's character cube) comprising eight dark profiles that can be used to compare individuals who differ in one dark character trait while holding the other two constant. Our aim was to investigate in which circumstances individuals who are high in each one of the dark character traits differ in Cloninger's "light" character traits: self-directedness, cooperativeness, and self-transcendence. We also investigated if people's dark character profiles were associated to their light character profiles.

Method: A total of 997 participants recruited from Amazon's Mechanical Turk (MTurk) responded to the Short Dark Triad and the Short Character Inventory. Participants were allocated to eight different dark profiles and eight light profiles based on their scores in each of the traits and any possible combination of high and low scores. We used three-way interaction regression analyses and t-tests to investigate differences in light character traits between individuals with different dark profiles. As a second step, we compared the individuals' dark profile with her/his character profile using an exact cell-wise analysis conducted in the ROPstat software (http://www.ropstat.com).

Results: Individuals who expressed high levels of Machiavellianism and those who expressed high levels of psychopathy also expressed low self-directedness and low cooperativeness. Individuals with high levels of narcissism, in contrast, scored high in self-directedness. Moreover, individuals with a profile low in the dark traits were more likely to end up with a profile high in cooperativeness. The opposite was true for those individuals with a profile high in the dark traits. The rest of the cross-comparisons revealed some of the characteristics of human personality as a non-linear complex dynamic system.

Conclusions: Our study suggests that individuals who are high in Machiavellianism and psychopathy share a unified non-agentic and uncooperative character (i.e., irresponsible, low in self-control, unempathetic, unhelpful, untolerant), while individuals high in narcissism have a more unique character configuration expressed as high agency and, when the other dark traits are high, highly spiritual but uncooperative. In other words, based on differences in their associations to the light side of character, the Dark Triad seems to be a dyad rather than a triad. 
63

64

65

66

67 "There's been an awakening. Have you felt it? The Dark side, and the Light."

68

69

70

71

72

73

74 75

From the movie Star Wars: The Force Awakens.

Dark Triad Theory (Furnham, Richards \& Paulhus, 2013) posits that people's malevolent Narcissism is the tendency to lack empathy, have fantasies of enormous power, beauty and exhibitionism (Raskin \& Hall, 1979). Psychopathy is expressed as low empathy, low anxiety, and high impulsive and thrill-seeking behavior (Hare, 1985). Nevertheless, whether the dark unification hypothesis) is still under debate. on each one of the dark traits also display different behaviors (Jones \& Figueredo, 2013; Hawley, are more likely to use strategic planning in their manipulations, whereas individuals high on psychopathy crave quick gratification and have problems with impulse control when they manipulate others to gain self-validation with no regard to who they might hurt in doing so character is represented by three dark traits: Machiavellianism, narcissism, and psychopathy (cf. Paulhus \& Williams, 2002). Machiavellianism is expressed as a personality characterized as cold, manipulative, and with a cynical worldview and lack of morality (Christie \& Geis, 1970). success, and at the same time have problems with criticism and show exploitativeness and traits are three distinctive traits (i.e., uniqueness hypothesis) or are one global trait (i.e.,

In line with the uniqueness hypothesis, research suggests that individuals who score high 2003). For example, while individuals who score high on either Machiavellianism or psychopathy can be defined as manipulative, individuals who score high on Machiavellianism manipulate others (Browner \& Price, 2001). Additionally, individuals high on narcissism tend to (Watson, Grisham, Trotter, \& Biderman, 1984). In other words, although all three dark traits can 
88 be defined as manipulative at the conceptual level, the specific manipulative behavior of 89 individuals who score high on each of the traits, is distinctive depending on which trait the 90 individual might score high on. Accordingly, these malevolent traits are related, but the 91 relationship is not strong, thus, the dark traits are suggested as independent from each other 92 (Paulhus \& Williams, 2002). Hence, this emphasizes that a person can be high on any of the 93 traits, while being low in the others, in turn, suggesting not only differences between individuals 94 but also within the individual. However, factor-analytic studies show, for example, that all three of the dark traits load on the HEXACO ${ }^{1}$ Honesty-Humility factor; suggesting that all three dark traits are negatively

97 related to peoples' levels of sincerity, fairness, greed avoidance, and modesty (Lee \& Ashton, 98 2005; see also Furnham \& Crump, 2005). In addition, Paulhus and Williams (2002) found that 99 individuals who scored high in any of the three Dark Triad traits scored low in the Big Five personality trait of agreeableness (i.e., the tendency to be kind, sympathetic, cooperative, warm, and considerate) and that individuals who scored high in psychopathy and narcissism also scored

102 high on extraversion (i.e., individual's tendency to be enthusiastic, action-oriented, outgoing and 103 enjoy interacting with people) and openness (i.e., the tendency to be open to new experiences, 104 inquisitive, and imaginative). Individuals high in Machiavellianism and psychopathy also score 105 low in self-discipline, lack sense of duty, and have difficulties to control, regulate, and direct 106 their impulses (i.e., low levels of the Big Five trait of conscientiousness). These associations are 107 in line with a unified view of the dark traits, that is, suggesting a common core (Jakobwitz \& 108 Egan, 2006; Lee \& Ashton, 2005; Paulhus \& Williams, 2002. See also Kajonius, Persson,

\footnotetext{
${ }^{1}$ The HEXACO model of personality structure is a six-dimensional model of human personality based on findings from a series of lexical studies involving several European and Asian languages. The six factors, or dimensions, include Honesty-Humility (H), Emotionality (E), Extraversion (X), Agreeableness (A), Conscientiousness (C), and Openness to Experience (O). Each factor is composed of traits with characteristics indicating high and low levels of the factor. Retrieved from https://en.wikipedia.org/wiki/HEXACO_model_of personality structure.
} 
109 Rosenberg, \& Garcia, 2015). Nevertheless, while some researchers have confirmed these results

110 using different samples (e.g., Lee \& Ashton, 2005), other researchers have not (e.g., Jakobwitz \&

111 Egan, 2006). In other words, even if there are some correlations between the Dark Triad and the

112 Big Five, these are neither large nor consistent (Vernon, Villani, Vickers \& Harris, 2008). The

113 only exception to this conclusion is the negative relationship between each of the dark traits and

114 agreeableness (e.g., Jakobwitz \& Egan, 2006; Lee \& Ashton, 2005; Paulhus \& Williams, 2002).

115 In sum, current research addressing distinctions in how people's dark character is related

116 to specific personality traits, such as, extraversion, show mixed and inconsistent results. Probably

117 because the personality models used to find differences or similarities between people's dark

118 character traits only represent individuals' emotional reactions or temperament (e.g., McAdams,

119 2001; Haidt, 2006). After all, temperament is not useful in the distinction of who ends up with a

120 mature or immature character (Cloninger, 2004). Indeed, not all individuals who are extroverts

121 end up scoring high in psychopathy and/or narcissism (i.e., antecedent variables have different

122 outcomes or "multi-finality") and high scores in each one of the dark traits might have different

123 antecedents (i.e., "equifinality")" (see Cloninger \& Zohar, 2011). In other words, it might be

124 inappropriate to assume linearity of effects in the context of personality (i.e., temperament

125 predicts character linearly or vice versa). We argue that in order to find individual differences

126 that can distinguish between peoples' malevolent tendencies, we need to use personality models

127 that cover aspects of human personality that actually represent what individuals make of

128 themselves intentionally or character.

129 Cloninger's personality model (Cloninger et al., 1993; Cloninger, 2004, 2007, 2013), for

130 instance, comprises three "light" character dimensions: self-directedness, cooperativeness, and

131 self-transcendence. Although Cloninger does not usually call these ternary model of character as 
132 "light", we argue that in contrast to the emotions derived from extreme expressions from our

133 temperament traits and probably even the dark side our character (e.g., joy, sadness, disgust, fear,

134 and anger), expressions from the character traits proposed by Cloninger are responsible for light

135 feelings, such as, feelings of hope in that we are capable to cope with life (i.e., self-directedness),

136 feelings of love for and from others (i.e., cooperativeness), and whether we feel connected to

137 something bigger than ourselves (i.e., self-transcendence) — that is, a ternary unity of the being:

138 body, mind, and psyche ${ }^{2}$. An individual high in self-directedness is reliable, strong, mature, goal-

139 oriented and self-sufficient; an individual high in cooperativeness is fair, tolerant, empathetic,

140 responsive to others needs, supportive and cooperative; and an individual high in self-

141 transcendence is spiritual, satisfied, patient, selfless and creative (Cloninger et al., 1993).

142 Cloninger has proposed that this ternary structure of character can be studied through eight

143 different character profiles, that is, all the possible combinations of people's high and low scores

144 in the character traits as measured by the Temperament and Character Inventory (e.g., Cloninger

$145 \&$ Zohar, 2011). The creation of the character profiles using the median as the reference point

146 allows the evaluation of the non-linear effect of each of the character traits on, for example, well-

147 being by comparing the effect of extremes (high vs. low) of each character trait when controlling

148 the other two. In other words, the advantage of studying multidimensional profiles of specific

149 combinations of traits allows the understanding of the experience in an individual who is

150 "adapting within his or her biopsychosocial context” (Cloninger \& Zohar, 2011, p.25).

151 In the present study, we used Cloninger's character cube as an analogy to investigate if

152 individuals' dark tendencies differ with respect to their self-concept or character (see Figure 1).

153 Specifically, our research question was: in which circumstances do individuals high in one dark

${ }^{2}$ Observe that the Greek word psyche found in psychology and psychiatry stands for "life, soul, or spirit,", which is distinct from soma, which refers to the "body" (Cloninger, 2004; see also Cloninger \& Cloninger, 2011ab; Cloninger, Salloum, \& Mezzich, 2012). 
154 trait express less/more self-directedness, cooperativeness and self-transcendence? We expected 155 that, if the Dark Triad constitutes a ternary structure of malevolent character traits, then the three 156 dark traits should be distinguishable in the individual's goals and values in relation to oneself 157 (i.e., self-directedness), others or society (i.e., cooperativeness), and something bigger than 158 oneself and society, for example, the universe, nature or/and God (i.e., self-transcendence). 159 Additionally, we also investigated if people with specific dark profiles were more likely to end 160 up with specific character profiles.

Figure 1 should be here

162

\section{Method}

\section{Ethical statement}

164 After consulting with the Network for Empowerment and Well-Being's Review Board we 165 arrived at the conclusion that the design of the present study (e.g., all participants' data were 166 anonymous and will not be used for commercial or other non-scientific purposes) required only 167 informed consent from the participants.

\section{Participants and procedure}

169 Participants $(N=1050)$ were recruited through Amazon's Mechanical Turk (MTurk; 170 www.mturk.com/mturk/welcome) (for validation of MTurk as a data collection tool see among 171 others Rand, 2011; Buhrmeister, Kwang \& Gosling, 2011). All participants were informed that

172 the survey was voluntary, anonymous, and that the participants could terminate the survey at any 173 time. The MTurk workers received 50 cents (US-dollars) as compensation for participating. Two 174 control questions were added to the survey, to control for automatic responses (e.g., "This is a 175 control question, please answer "neither agree or disagree"). After taking away those who 176 responded erroneously to one or both of the control questions $(n=53,5.31 \%$ of all who 
177 participated), the final sample constituted 997 participants, 362 males (36.31\%) and 630 females

$178(63.19 \%)$, with an age mean $=34.13$ years, $S D=12.37$.

179 Instruments

180 The Short Character Inventory. This instrument was originally designed by C. R. Cloninger for 181 Time Magazine as a short version of the 238-item Temperament and Character Inventory 182 (Cloninger et al., 1993). It has never been tested empirically, but the items are all imbedded in 183 the larger version. This makes it a brief version that is easy to administer for testing relationships 184 among personality variables in large groups. Permission was obtained from C. R. Cloninger in 185 order to include it in the present study. The inventory contains 15 items, 5 per dimension, rated 186 on a 5-point Likert scale $(1=$ definitely false, $5=$ definitely true). Examples of the items are:

187 "Each day I try to take another step toward my goals" (self-directedness; Cronbach's $\alpha=.56$ ), "I 188 enjoy getting revenge on people who hurt me" (cooperativeness, reversed item, Cronbach's $\alpha=$ 189 .54), and "Sometimes I have felt like I was part of something with no limits or boundaries in time 190 and space" (self-transcendence, Cronbach's $\alpha=.57$ ).

192 items, 9 per each dark trait. Examples of the items are: "Most people can be manipulated" 193 (Machiavellianism, Cronbach's $\alpha=.76$ ), "People see me as a natural leader" (narcissism; 194 Cronbach's $\alpha=.74$ ), and "Payback needs to be quick and nasty" (psychopathy; Cronbach's $\alpha=$ 195 .73). The items were rated on 5-point Likert scale $(1=$ strongly disagree, $5=$ strongly agree $)$.

\section{Statistical procedure}

197 The sample was divided into subjects above (high) and below (low) the median for each of the 198 three dark traits: Machiavellianism (median =3.00; $\mathrm{M}$ for high, $\mathrm{m}$ for low), narcissism (median $199=2.67 ; \mathrm{N}$ for high, $\mathrm{n}$ for low), and psychopathy (median $=1.78 ; \mathrm{P}$ for high, $\mathrm{p}$ for low). Then the 
200 participants were grouped according to all the possible combinations of high and low dark trait

201 scores to define the eight possible Dark Triad profiles: MNP "maleficent" $(n=232,23.3 \%)$,

202 MNp "manipulative narcissistic" $(n=66,6.6 \%), \mathrm{MnP}$ "anti-social” ( $n=134,13.4 \%)$, Mnp

203 "Machiavellian" $(n=92,9.2 \%), \mathrm{mNP}$ "psychopathic narcissistic" $(n=86,8.6 \%), \mathrm{mNp}$

204 "narcissistic" $(n=93,9.3 \%), \mathrm{mnP}$ "psychopathic" $(n=76,7.6 \%)$, and mnp "agreeable" $(n=$ $205218,22.0 \%$ ). See Figure 2.

206

Figure 2 should be here

207

We followed the same procedure using participants' character scores. The sample was 208 divided into subjects above (high) and below (low) the median for each of the three character traits: self-directedness (median $=3.60 ; \mathrm{S}$ for high, $\mathrm{s}$ for low), cooperativeness (median = 3.80; $\mathrm{C}$

210 for high, $\mathrm{c}$ for low), and self-transcendence (median $=3.00$; $\mathrm{T}$ for high, $\mathrm{t}$ for low). Then the 211 participants were grouped according to all the possible combinations of high and low character 212 trait scores to define the eight possible light character profiles: SCT "creative" $(n=149,14.9 \%)$,

213 SCt "organized" $(n=144,14.4 \%)$, ScT "fanatical” $(n=73,7.3 \%)$, Sct "autocratic" $(n=94,9$. 214 4\%), sCT “moody" ( $n=103,10.3 \%)$, sCt "dependent” $(n=98,9.8 \%)$, scT "disorganized” $(n=$ $215137,13.7 \%)$, and sct "depressive" $(n=199,20.0 \%)$.

\section{Results and discussion}

217 Table 1 shows the correlations, means, standard deviation, and Cronbach's alpha for each of the 218 character traits. The significant correlations between the light character traits was relatively low 219 and varied between $r=.10$ (self-directedness and self-transcendence) and .29 (self-directedness 220 and cooperativeness), while the significant correlations between the dark character traits varied 221 between $r=.35$ (Machiavellianism and narcissism) and .51 (Machiavellianism and 
222 psychopathy). The significant correlations between dark and light character traits varied between

$223 r=.14$ (self-directedness and narcissism) and -.58 (cooperativeness and psychopathy).

224

225

226

227

228

229

230

231

232

233

234

235

236

237

238

239

240

241

242

243

\section{Table 1 should be here}

Despite the fact that earlier studies using light character profiles (Cloninger \& Zohar, 2011) have used t-tests to address the question of in which circumstances do individuals high in one trait express less/more of an outcome variable, we opted to first conduct three three-way interaction regression analyses using the whole scale range of the dark character traits as the predictor variables and the light character traits as the outcome. This strategy, although linear, allowed us to have higher statistical power. All variables were standardized before performing the analysis. The first model explained $19.30 \%$ of the variance in self-directedness, the second model explained $44.30 \%$ of the variance in cooperativeness, and the third model explained only $4.60 \%$ of the variance in self-transcendence.

In step 1, Machiavellianism was negatively associated to all three light character traits while narcissism was positively associated to all three light character traits. Psychopathy was negatively associated to cooperativeness and self-transcendence. In step 2, there was a small but significant negative interaction effect between Machiavellianism and psychopathy on cooperativeness and a small but significant positive interaction effect between Narcissism and psychopathy on cooperativeness. In step 3, however, there was no significant three-way interaction effect between the dark character traits in relation to any of the light character traits. See Table 2 for the details. Nevertheless, seeing that we based our investigation on dark profiles, analogical to Cloninger's light character profiles, rather than linear analyses we continued with comparison analyses between dark profiles.

Table 2 should be here 
Paired t-tests were performed to evaluate the non-linear influence of each of the Dark

246 Triad profiles on the character traits. The comparisons investigated the effect of extremes of each

247 Dark Triad profile when the other two were held constant (see Table 3 for the details).

248 Individuals high in Machiavellianism scored, in all cases, lower in self-directedness and

249 cooperativeness than individuals low in Machiavellianism. Thus, suggesting a clear and unique

250 association between Machiavellian tendencies and low levels of self-acceptance, sense of

251 autonomy and responsibility, self-control, and self-actualization (i.e., low self-directedness), and

252 also low tolerance towards others, unhelpfulness, and low levels of empathy (i.e., low

253 cooperativeness). Individuals high in Machiavellianism, compared to those low in

254 Machiavellianism, scored lower in self-transcendence only when they were low in narcissism

255 and high in psychopathy (MnP vs. mnP).

256 Individuals high in narcissism were, in all cases, higher in self-directedness than

257 individuals low in narcissism. That is, in contrast to its negative relation to both

258 Machiavellianism and psychopathy, self-directedness is positively associated to narcissism. This

259 suggests that narcissism is distinctive from the other two traits when it comes to agentic or self-

260 directed behavior. Moreover, individuals high in narcissism, compared to those low in

261 narcissism, reported lower cooperativeness only when they were low in both Machiavellianism

262 and psychopathy (mNp vs. mnp). This suggests that narcissism in its pure form is associated with

263 unhelpfulness, low tolerance towards others, and low empathy. Finally, individuals high in

264 narcissism, compared to those low in narcissism, reported higher self-transcendence only when

265 they were high in both Machiavellianism and psychopathy (MNP vs. MnP). This suggests that an

266 individual who is high in the three dark traits might be goal-directed and unempathic (i.e., high

267 self-directedness and low cooperativeness). At the same time she/he might have a sense of being 
268 in connection with something divine or universal (i.e., high self-transcendence). In other words,

269 the "maleficent" dark profile (i.e., MNP) seems to correspond to a "fanatical" light character

270 profile (i.e., ScT) — individuals with a "fanatical" profile can be characterized as independent and

271 paranoid, and being projective of blame (Cloninger, Bayon \& Svrakic, 1998). This is actually in

272 accordance to descriptions of successful dictators such as Gadhafi and Saddam or terrorists such

273 as Osama Bin Laden and Anders Breivik who have been suggested to have a "maleficent" dark

274 character profile (i.e., MNP; see Furnham, Richards \& Paulhus, 2013).

275 Individuals high in psychopathy were, in all cases, lower in self-directedness and

276 cooperativeness when compared to individuals low in psychopathy. Thus, as for

277 Machiavellianism, this suggests a clear and unique association between psychopathy and low

278 self-directed behavior and uncooperativeness. Individuals high in psychopathy, compared to

279 those low in psychopathy, reported lower self-transcendence only when they were high in

280 Machiavellianism and low in narcissism (MnP vs. Mnp).

281

Table 3 should be here

282

In the second analysis, we compared the individual's dark profile with her/his character

283 profile using an exact cell-wise analysis in the ROPstat software (Vargha, Torma \& Bergman,

284 2015; http://www.ropstat.com). The aim with this base model was to create a reference (i.e., an

285 estimated expected cell frequency) to which we compared the observed cell frequency (see von

286 Eye, Bogat \& Rhodes, 2006). In short, if a specific cell contains more cases than expected under

287 this base model, this cell indicates a relationship that exists only in this particular sector of the

288 cross-classification, that is, it constitutes a type. If a cell, in contrast contains fewer cases than

289 expected under the base model, this cell also indicates a local relationship, that is, it constitutes 
290 an antitype (see also Bergman \& El-Khouri, 1987). We examined the idea of dark profile having

291 an effect on character profile membership (see Table 4).

292 For individuals with an "agreeable" dark profile (i.e., mnp: low in all dark traits) there

293 was a higher probability (i.e., type) to end up with a high cooperative profile (i.e., SCT, SCt,

$294 \mathrm{sCT}, \mathrm{sCt}$ ) and a lower probability (i.e., anti-type) of ending up with a low cooperative profile

295 (i.e., ScT, Sct, scT, Sct). As it could be expected, the opposite was found for the "maleficent"

296 dark profile (i.e., MNP: high in all dark traits). This is in line with research linking Machiavellian

297 tendencies and dark tendencies per se to low agreeableness and unhelpful behavior (i.e., the

298 unification argument). For the rest of the profiles, our analyses show a complex pattern of

299 possible combinations. For example, although individuals with an "anti-social" dark profile (i.e.,

$300 \mathrm{MnP}$ ) were less likely (i.e., anti-type) to end up with a high cooperative character profile, they

301 were more likely (i.e., type) to end up with either a "disorganized" (i.e., scT) or a "depressive"

302 character profile (i.e., sct). In other words, all individuals with an "anti-social" dark profile are

303 uncooperative, while some of them are at the same time either highly spiritual or with

304 underdeveloped character. Additionally, as observed in the t-tests analyses, the "fanatical" light

305 character profile (i.e., ScT) was common (i.e., type) for those who had a "maleficent" dark

306 character profile (i.e., MNP) and less common (i.e., anti-type) for those who had an "agreeable"

307 dark profile (i.e., mnp). See Table 4.

308

$\underline{\text { Table } 4 \text { should be here }}$

309 Limitations

310 The data is self-reported and therefore subject to personal perceptual bias. Moreover, the light

311 character scales showed low reliabilities, probably because the instrument has so few items. But

312 the items are all imbedded in the larger version, which has been validated across many studies. 
313 This makes it a brief version that is easy to administer for testing relationships among personality

314 variables in large groups of subjects but probably not for precise assessment of individuals.

315 Nevertheless, given the observation of the low Cronbach's alpha scores, it seems appropriate

316 that the factorial validity should be examined in future studies. In that case, researchers should

317 consult suggestions regarding the evaluation of short questionnaires (e.g., Marsh, Martin, \&

318 Jackson, 2010; Olaru, Witthöft \& Wilhelm, 2015). That being said, this short version actually

319 discerned the expected patterns among the Dark Triad profiles. The question of causality is,

320 however, beyond the present cross-sectional study.

321 Some aspects related to the use of MTurk as a data collection method might influence the

322 validity of the results, such as, workers' attention levels, cross-talk between participants, and the

323 fact that participants get remuneration for their answers (Buhrmester, Kwang \& Gosling, 2011).

324 Nevertheless, a large quantity of studies show that data on psychological measures collected

325 through MTurk meets academic standards, is demographically diverse, and also that health

326 measures show satisfactory internal as well as test-retest reliability (Buhrmester, Kwang \&

327 Gosling, 2011; Horton, Rand \& Zeckhauser, 2011; Shapiro, Chandler \& Mueller, 2013; Paolacci,

328 Chandler \& Ipeirotis, 2010). In addition, the amount of payment does not seem to affect data

329 quality; remuneration is usually small, and workers report being intrinsically motivated (e.g.,

330 participate for enjoyment) (Buhrmester, Kwang \& Gosling, 2011).

331 Finally, it is plausible to argue that dichotomizing into groups that are classified as being

332 low or high on traits will likely lead to a loss of power effectively equivalent to a loss of sample

333 size (e.g., MacCallum, Zhang, Preacher \& Rucker, 2002). Additionally, since median splits

334 distort the meaning of high and low, it is plausible to criticize the validity of this approach to

335 create the profiles - scores just-above and just-below the median become high and low by 
336 arbitrariness, not by reality (Schütz, Archer \& Garcia, 2013; Garcia, MacDonald, \& Archer,

337 2015). In light of these arguments, we included as a first step the three three-way interaction

338 regression analyses, which actually confirmed the core findings. However, others have argued

339 that from a person-centered framework personality dimensions within the individual can be seen

340 as interwoven components with whole-system properties (cf. Bergman \& Wångby, 2014). The

341 outlook of the individual as a whole-system unit is then best studied by analyzing patterns of 342 information or profiles (Bergman \& Wångby, 2014). Although at a theoretical level there is a

343 myriad of probable patterns of combinations of peoples' levels of personality traits, if viewed at

344 a global level, there should be a small number of more frequently observed patterns or "common

345 types” (Bergman \& Wångby, 2014; Bergman \& Magnusson, 1997; see also Cloninger, Svrakic

$346 \&$ Svrakic, 1997, who explain nonlinear dynamics in complex adaptive systems). It is beyond the

347 scope of the present study to discern the best possible way to arrive to the dark profiles but

348 researchers should definitely consider putting the parts together. After all, although all possible

349 variations of personality profiles is large, human personality is a non-linear dynamic system

350 (Cloninger, 2004) that responds to the laws of attractor states ${ }^{3}$, which are essential for the

351 understanding of most physical and human phenomena (Hiver, 2014; see also Prigogine \&

352 Stengers, 1984). Here we based our choice of using median splits in two presuppositions: (1) that

353 at the conceptual and theoretical level the dark character traits constitute a ternary structure of

354 human dark/maladaptive character and (2) that an opposite ternary structure of human

355 light/adaptive character generates eight possible profile combinations that have been largely

356 studied using median splits. The limitation of our approach might actually reside in the fact that

357 the Dark Triad is not a ternary structure as the one represented by the light character traits in

\footnotetext{
${ }^{3}$ Fixed points, or steady states of a given dynamical system; these are values of the variable that don't change over time. Some of these fixed points are attractive, meaning that if the system starts out in a nearby state, it converges towards the fixed point (https://en.wikipedia.org/wiki/Dynamical_systems_theory).
} 
358 Cloninger's biopsychosociopiritual model of personality. Indeed, the comparison of light

359 character profiles with the dark ones shows that the dark triad inadequately captures the anti360 types already measured well by the light triad.

\section{Conclusions and final remarks}

362 Far from the mixed patterns using the Big Five traits (e.g., Jakobwitz \& Egan, 2006; Paulhus \&

363 Williams, 2002), this study suggest that Machiavellianism and psychopathy share a unified but

364 unique non-agentic (low self-directedness) and uncooperative (low cooperativeness) character;

365 while narcissism has a unique character configuration expressed as high agency. In other words, 366 the Dark Triad does not seem to represent a ternary structure as Cloninger's character model, it 367 rather is a dyad of malevolent character traits in relation to the self (i.e., narcissism) and others 368 (i.e., both Machiavellianism and Psychopathy). Specifically, the Dark Triad lacks a dark trait that 369 corresponds uniquely to a spiritual dimension of human character. Recently, however, Paulhus 370 (2014) has suggested everyday sadism as a fourth component, making the triad into a tetrad. It is 371 plausible that, future studies might find that enjoyment of cruelty against other human beings and 372 animals, is uniquely associated to the inability of transcend the self and feel part of the whole 373 universe.

374 "One of the most highly developed skills in contemporary Western civilization is dissection: the 375 split-up of problems into their smallest possible components. We are good at it. So good, we 376 often forget to put the pieces back together again.

377 This skill is perhaps most finely honed in science. There we not only routinely break 378 problems down into bite-sized chunks and mini-chunks, we then very often isolate each one from 379 its environment by means of a useful trick. We say ceteris paribus — all other things being equal. 
380 In this way we can ignore the complex interactions between our problem and the rest of the 381 universe."

382 Alvin Toffler in the Foreword to Order out of Chaos: Man's New Dialogue with Nature by Ilya 383 Prigogine and Isabelle Stengers (1984).

384

385

386

387

388

389

390

391

392

393

394

395

396

397

398

399

400

401

402

\section{References}

Bergman, L. R., \& El-Khouri, B. (1987). EXACON: A Fortran 77 program for the exact analysis of single cells in a contingency table. Educational and Psychological measurement, 47(1), 155-161. DOI 10.1177/0013164487471024.

Bergman, L. R., \& Magnusson, D. (1997). A person-oriented approach in research on developmental psychopathology. Development and Psychopathology, 9(2), 291- 319. DOI: 10.1017/S095457949700206X.

Bergman, L. R., \& Wångby, M. (2014). The person-oriented approach: A short theoretical and practical guide. Eesti Haridusteaduste Ajakiri, 2, 29-49. Doi: 10.12697/eha.2014.21.02b.

Brower, M. C., \& Price, B. H. (2001). Neuropsychiatry of frontal lobe dysfunction in violent and criminal behaviour: A critical review. Journal of Neurology, Neurosurgery \& Psychiatry, $71,720-726$

Buhrmester M. D., Kwang T., \& Gosling S. D. (2011). Amazon's Mechanical Turk: a new source of inexpensive, yet high-quality, data? Perspectives on Psychological Science, 6, 3-5

Christie, R., \& Geis, F. L. (1970). Studies in Machiavellianism. New York: Academic Press.

Cloninger, C. R. (2004). Feeling good: The science of well-being. New York: Oxford University Press

Cloninger, C. R. (2007). Spirituality and the science of feeling good. Southern Medical Journal, $100,(7), 740-3$ 
403 Cloninger, C. R. (2013). What makes people healthy, happy, and fulfilled in the face of current $404 \quad$ world challenges. Mens Sana Monographs, 11, 16-24.

405 Cloninger, C. R., Bayon, C.,\& Svrakic, D. M. (1998). Measurement of temperament and 406 character in mood disorders: a model of fundamental states as personality types, Journal of 407 Affective Disorders, 51, 21-32.

408 Cloninger, C. R., \& Cloninger, K. M. (2011a). Person-centered Therapeutics. The International 409 Journal of Person Centered Medicine, 1 (1), 43-52.

410 Cloninger, C. R., \& Cloninger, K. M. (2011b). Development of Instruments and Evaluative 411 Procedures on Contributors to Illness and Health. The International Journal of Person $412 \quad$ Centered Medicine, 1 (3), 446-455.

413 Cloninger, C. R., Salloum, I. M., \& Mezzich, J. E. (2012). The Dynamic Origins of Positive $414 \quad$ Health and Wellbeing. The International Journal of Person Centered Medicine, 2 (2), 179$415 \quad 187$.

416 Cloninger, C. R., Svrakic, D. M., \& Przybeck, T. R. (1993). A psychobiological model of 417 temperament and character. Archives of General Psychiatry, 50(12), 975-990.

418 Cloninger, C. R., Svrakic, N. M., \& Svrakic, D. M. (1997). Role of personality self-organization 419 420 in development of mental order and disorder. Development and Psychopathology, 9, 881906.

421 Cloninger, C.R., \& Zohar, A.H (2011). Personality and the perception of health and happiness. 422 Journal of Affect Disorder, 128(1-2): 24-32.

423 Furnham, A., \& Crump, J. (2005). Personality traits, types, and disorders: An examination of the 424 relationship between three self-report measures. European Journal of Personality, 19, 167 $425 \quad 184$. 
426 Furnham, A., Richards, S. C., \& Paulhus, D. L. (2013). The Dark Triad of Personality: A 10

427 Year Review. Social and Personality Psychology Compass 7/3, 199-216.

428 Garcia, D., MacDonald, S., \& Archer, T. (2015). Two Different Approaches to The Affective 429 Profiles Model: Median Splits (Variable-Oriented) and Cluster Analysis (Person-Oriented). PeerJ 3:e1380. DOI: 10.7717/peerj.1380.

431 Haidt, J. (2006). The happiness hypothesis: Finding modern truth in ancient wisdom. New York: 432 Basic Books.

Hare, R. D. (1985). Comparison of procedures for the assessment of psychopathy. Journal of 434 Consulting and Clinical Psychology, 53, (1), 7-16

435

436

437

438

439

440

441

442

443

444

445

446

447

Hawley, P. (2003). Prosocial and coercive configurations of resource control in early adolescence: A case for the well-adapted Machiavellian. Journal of Developmental Psychology, 49, 279-309.

Hiver, P. (2014). Attractor States. In Z. Dornyei, P. MacIntyre, \& A. Henry (Eds.), Motivational Dynamics in Language Learning. Bristol: Multilingual Matters (pp. 20-28).

Jakobwitz, S., \& Egan, V. (2006). The dark triad and normal personality traits. Personality and Individual Differences, 2, 331-339.

Jones, D. N., \& Figueredo, A. J. (2013). The core of darkness: Uncovering the heart of the Dark Triad. European Journal of Personality, 27, 521-531.

Jones, D. J., \& Paulhus, D. L. (2014). Introducing the Short Dark Triad (SD3): A Brief Measure of Dark Personality Traits. Assessment, 21, (1), 28-41.

Kajonius, P. J., Persson, B. N., Rosenberg, P. \& Garcia, D. (2015). The (mis)measurement of the Dark Triad Dirty Dozen: Exploitation at the core of the scale. PeerJ PrePrints $3: \mathrm{e} 1605 v 1$. 
448 Lee, K., \& Ashton, M. C. (2013). Prediction of self- and observer report scores on HEXACO-60 449 and NEO-FFI scales. Journal of Research in Personality, 47, 668-675.

450 MacCallum, R. C., Zhang, S., Preacher, K. J., \& RUCker, D. D. (2002). On the practice of 451 dichotomization of quantitative variables. Psychological Methods, 7, 19-40. DOI: 452 10.1037//1082-989X.7.1.19.

453

454

455

456

457

458

459

460

461

462

463

464

465

466

467

468

469

Marsh, H.W., Martin, A.J., \& Jackson, S. (2010). Introducing a short version of the Physical Self-Description Questionnaire: New strategies, short-form evaluative criteria, and applications of factor analyses. Journal of Sport and Exercise Psychology, 32, 438-482.

McAdams, D. P. (2001). The psychology of life stories. Review of General Psychology, 5, 100122.

Olaru, G., Witthöft, M., \& Wilhelm, O. (2015). Methods Matter: Testing Competing Models for Designing Short-scale Big-Five Assessments. Journal of Research in Personality, 59, 56-68.

Paulhus, D.L. (2014). Toward a taxonomy of dark personalities. Current Directions in Psychological Science, 23, 421-426.

Paulhus D. L., \& Williams, K. M. (2002). The Dark Triad of personality: Narcissism, Machiavellianism, and psychopathy. Journal of Research in Personality, 36, 556-563.

Prigogine, I., \& Stengers, I. (1984). Order out of chaos: Man's new dialogue with nature. New York: Bantam Books, Inc.

Rand, D. G. (2011). The promise of Mechanical Turk: How online labor markets can help theorists run behavioral experiments. Journal of Theoretical Biology, 299, 172-179

Raskin, R., \& Hall. C. S. (1979). A Narcissistic Personality Inventory. Psychological Reports, 45,590 . 
470 Schütz, E., Garcia, D., \& Archer, T. (2014). Affective state, stress, and type a-personality as a 471 function of gender and affective profiles. International Journal Of Research Studies in $472 \quad$ Psychology, 3, 51-64. DOI 10.5861/ijrsp.2013.450.

473 Vargha, A., Torma, B., Bergman, L. R. (2015). ROPstat: A general statistical package useful for 474 conducting person-oriented analyses. Journal of Person-Oriented Research, 1, 87-97. DOI: $475 \quad$ 10.17505/jpor.2015.09.

476 Vernon, P. A., Villani, V. C., Vickers, L. C., \& Harris, J. A. (2008). A behavioural genetic 477 investigation of humour styles and their correlations with the Big-5 personality dimensions. $478 \quad$ Personality and Individual Differences, 44, 1116-1125.

479 von Eye, A., \& Bogat, G. A., \& Rhodes, J. E. (2006). Variable-oriented and person-oriented 480 perspectives of analysis: The example of alcohol consumption in adolescence. Journal of 481 adolescence, 29, 981-1004. DOI 10.1016/j.adolescence.2006.06.007.

482 Watson, P. J., Grisham, S. O., Trotter, M. V., \& Biderman, M. D. (1984). Narcissism and 483 empathy: validity evidence for the Narcissistic Personality Inventory. Journal of Personality

484 Assessment, 48, 301-305.

485

486

487

488

489 Legends

490 Figure 1. The Dark Cube as an analogy to Cloninger's character cube, showing all eight possible 491 combinations of high/low scores in Machiavellianism, narcissism, and psychopathy. 
492 Note: adapted with permission from C. R. Cloninger. The directions of the arrows represent 493 higher values. $\mathrm{M}=$ high Machiavellianism; $\mathrm{m}=$ low Machiavellianism; $\mathrm{N}=$ high narcissism; $\mathrm{n}=$ 494 low narcissism; $\mathrm{P}=$ high psychopathy; $\mathrm{p}=$ low psychopathy. Observe that the scale for 495 neuroticism does not match that of Figure 1 due to technical issues.

496 Figure 2. Distribution of the different dark character profiles in the Dark Cube.

497 Note: $\mathrm{M}=$ high Machiavellianism; $\mathrm{m}=$ low Machiavellianism; $\mathrm{N}=$ high narcissism; $\mathrm{n}=$ low 498 narcissism; $\mathrm{P}=$ high psychopathy; $\mathrm{p}=$ low psychopathy.

499 Table 1. The correlation matrix including means, standard deviations (SD) and Alphas of both 500 light and dark character traits.

501 Note: $* p<.05 ; * * p<.01 ; * * * p<.001$.

502 Yellow fields: significant correlations between light character traits.

503 Black fields: significant correlations between dark character traits.

504 Grey fields: significant correlations between light and dark character traits.

505 Table 2. Three-way interaction regression analyses using the Dark Triad traits (i.e., 506 Machiavellianism, narcissism, and Psychopathy) as the independent variables and the Light 507 Triad traits (i.e., self-directedness, cooperativeness and self-transcendence) as the outcome.

508 Note: $* p=.01, * * p<.05, * * * p=.000$.

509 Table 3. Results from the t-tests analyses for each Dark Triad character trait for self-directedness, 510 cooperativeness, and self-transcendence. Significant results are marked in bold type.

511 Note: $r_{p b}=$ point-biseral coefficient .

512 Table 4. Exact cell-wise analysis of two-way frequencies: dark and light character profiles.

513 Note: $* * * p<.001, * p<.05$.

514 TYPE: the observed cell frequency is significantly greater than the expected (grey fields). 
515 ANTI-TYPE: the observed cell frequency is significantly smaller than the expected (black

516 fields).

517 -: the observed cell frequency is as expected (white fields). 


\section{Table $\mathbf{1}$ (on next page)}

The correlation matrix including means, standard deviations (SD) and Alphas of both light and dark character traits.

Note: $* p<.05 ;{ }^{* *} p<.01 ; * * * p<.001$. Yellow fields: significant correlations between light character traits. Black fields: significant correlations between dark character traits. Grey fields: significant correlations between light and dark character traits. 
1 Table 1. The correlation matrix including means, standard deviations (SD) and Alphas of both light and dark character traits.

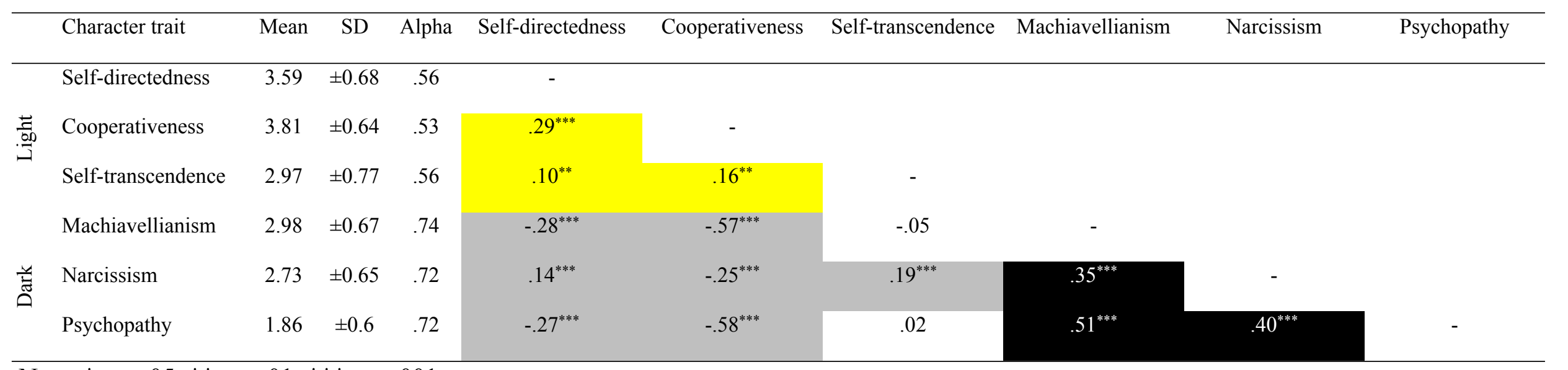

2 Note: $* p<.05 ; * * p<.01 ; * * * p<.001$

3 Yellow fields: significant correlations between light character traits.

4 Black fields: significant correlations between dark character traits.

5 Grey fields: significant correlations between light and dark character traits. 


\section{Table 2 (on next page)}

Three-way interaction regression analyses

Analyses using the Dark Triad traits (i.e., Machiavellianism, narcissism, and Psychopathy) as the independent variables and the Light Triad traits (i.e., self-directedness, cooperativeness and self-transcendence) as the outcome. Note: $* p=.01,{ }^{* *} p<.05,{ }^{* * *} p=.000$. 
1 Table 2. Three-way interaction regression analyses using the Dark Triad traits (i.e.,

2 Machiavellianism, narcissism, and Psychopathy) as the independent variables and the Light

3 Triad traits (i.e., self-directedness, cooperativeness and self-transcendence) as the outcome.

\begin{tabular}{|c|c|c|c|c|c|c|}
\hline \multirow[t]{2}{*}{ Variable } & \multicolumn{2}{|c|}{ Self-directedness } & \multicolumn{2}{|c|}{ Cooperativeness } & \multicolumn{2}{|c|}{ Self-transcendence } \\
\hline & $B$ & $\Delta R^{2}$ & $B$ & $\Delta R^{2}$ & $B$ & $\Delta R^{2}$ \\
\hline Step 1 & & .05 & & .44 & & .19 \\
\hline Machiavellianism (M) & $-.13 * *$ & & $-.38 * * *$ & & $-.27 * * *$ & \\
\hline Narcissism (N) & $.24 * * *$ & & $.06^{* *}$ & & $.35 * * *$ & \\
\hline Psychopathy (P) & -.01 & & $-.42 * * *$ & & $-.27 * * *$ & \\
\hline Step 2 & & .00 & & .00 & & .00 \\
\hline Machiavellianism & $-.13 * *$ & & $-.38 * * *$ & & $-.26 * * *$ & \\
\hline Narcissism & $.24 * * *$ & & .05 & & $.34 * * *$ & \\
\hline Psychopathy & .01 & & $-.42 * * *$ & & $-.27 * * *$ & \\
\hline Interaction $\mathrm{M}^{*} \mathrm{~N}$ & .04 & & .01 & & .05 & \\
\hline Interaction $\mathrm{M}^{*} \mathrm{P}$ & -.05 & & $-.05 * *$ & & -.01 & \\
\hline Interaction $\mathrm{N} * \mathrm{P}$ & -.01 & & $.06^{* *}$ & & -.01 & \\
\hline Step 3 & & .00 & & .00 & & .00 \\
\hline Machiavellianism & $-.14 * * *$ & & $-.39 * * *$ & & $-.26 * * *$ & \\
\hline Narcissism & $.22 * * *$ & & .04 & & $.35 * * *$ & \\
\hline Psychopathy & .01 & & $-.42 * * *$ & & $-.27 * * *$ & \\
\hline Interaction $\mathrm{M}^{*} \mathrm{~N}$ & .04 & & .02 & & .05 & \\
\hline Interaction $\mathrm{M} * \mathrm{P}$ & -.06 & & $-.06 * *$ & & .00 & \\
\hline Interaction $\mathrm{N} * \mathrm{P}$ & -.04 & & .05 & & -.00 & \\
\hline Interaction $\mathrm{M}^{*} \mathrm{~N} * \mathrm{P}$ & .03 & & .01 & & -.01 & \\
\hline Total & & .05 & & .44 & & .19 \\
\hline
\end{tabular}

4 Note: $* p=.01, * * p<.05, * * * p=.000$. 


\section{Table 3(on next page)}

Results from the t-tests analyses for each Dark Triad character trait for selfdirectedness, cooperativeness, and self-transcendence. Significant results are marked in bold type.

Note: $r_{p b}=$ point-biseral coefficient . 
1 Table 3. Results from the t-tests analyses for each Dark Triad character trait for self-directedness, cooperativeness, and self-

2 transcendence. Significant results are marked in bold type.

\begin{tabular}{|c|c|c|c|c|c|c|c|c|c|c|c|c|}
\hline & \multicolumn{4}{|c|}{ Self-directedness } & \multicolumn{4}{|c|}{ Cooperativeness } & \multicolumn{4}{|c|}{ Self-transcendence } \\
\hline & $t$ & $p$ & Cohen's d & $r_{p b}$ & $t$ & $p$ & $\begin{array}{c}\text { Cohen's } \\
d\end{array}$ & $r_{p b}$ & $t$ & $p$ & $\begin{array}{c}\text { Cohen's } \\
d\end{array}$ & $r_{p b}$ \\
\hline \multicolumn{13}{|c|}{ Machiavellianism } \\
\hline $\begin{array}{l}\text { MNP vs } \\
\text { mNP }\end{array}$ & -3.56 & .000 & -0.53 & .26 & -7.60 & .000 & -0.86 & .39 & -1.91 & .057 & -0.21 & .11 \\
\hline MNp vs mNp & -2.01 & .046 & -0.32 & .16 & -2.70 & .008 & -0.51 & .25 & 0.86 & .391 & 0.14 & .07 \\
\hline MnP vs mnP & -3.05 & .003 & -0.42 & .21 & -6.23 & .000 & -0.91 & .41 & -2.87 & .004 & -0.40 & .20 \\
\hline $\begin{array}{l}\text { Mnp vs mnp } \\
\text { Narcissism }\end{array}$ & -3.13 & .002 & -0.36 & .18 & -6.05 & .000 & -1.01 & .45 & 0.24 & .813 & 0.03 & .01 \\
\hline MNP vs MnP & 3.56 & .000 & 0.37 & .18 & -0.84 & .401 & -0.09 & .04 & 3.73 & .000 & 0.39 & .19 \\
\hline MNp vs Mnp & 2.60 & .010 & 0.42 & .20 & 0.56 & .575 & 0.09 & .04 & 1.87 & .063 & 0.30 & .15 \\
\hline mNP vs mnP & 2.73 & .007 & 0.43 & .21 & 0.25 & .803 & 0.04 & .02 & 1.42 & .158 & 0.22 & .11 \\
\hline $\begin{array}{l}\text { mNp vs mnp } \\
\text { Psychopathy }\end{array}$ & 2.79 & .006 & 0.39 & .19 & -2.38 & .018 & -0.27 & .13 & 1.43 & .153 & 0.16 & .08 \\
\hline $\begin{array}{l}\text { MNP vs } \\
\text { MNp }\end{array}$ & -3.23 & .001 & -0.38 & .18 & -7.86 & .000 & -0.91 & .42 & -1.42 & .157 & -0.17 & .08 \\
\hline MnP vs Mnp & -2.94 & .004 & -0.39 & .19 & -6.71 & .000 & -0.90 & .41 & -1.98 & .049 & -0.26 & .13 \\
\hline $\mathrm{mNP}$ vs $\mathrm{mNp}$ & -2.66 & .009 & -0.40 & .20 & -4.05 & .000 & -0.66 & .31 & 1.20 & .233 & 0.18 & .09 \\
\hline mnP vs mnp & -2.96 & .003 & -0.35 & .17 & -7.32 & .000 & -0.86 & .39 & 1.20 & .232 & 0.19 & .10 \\
\hline
\end{tabular}

3 Note: $r_{p b}=$ point-biseral coefficient. 


\section{Table 4 (on next page)}

Exact cell-wise analysis of two-way frequencies: dark and light character profiles.

Note: $* * * p<.001, * p<.05$. TYPE: the observed cell frequency is significantly greater than the expected (grey fields). ANTI-TYPE: the observed cell frequency is significantly smaller than the expected (black fields). -: the observed cell frequency is as expected (white fields). 
1 Table 4. Exact cell-wise analysis of two-way frequencies: dark and light character profiles.

\begin{tabular}{|c|c|c|c|c|c|c|c|c|c|}
\hline \multirow{2}{*}{ 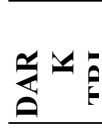 } & & \multicolumn{8}{|c|}{ CHARACTER PROFILE } \\
\hline & & $\begin{array}{c}\text { SCT } \\
\text { "Creative" }\end{array}$ & $\begin{array}{c}\text { SCt } \\
\text { "Organized" }\end{array}$ & $\begin{array}{c}\text { ScT } \\
\text { "Fanatical" }\end{array}$ & $\begin{array}{c}\text { Sct } \\
\text { "Autocratic" }\end{array}$ & $\begin{array}{c}\text { sCT } \\
\text { "Moody" } \\
\end{array}$ & $\begin{array}{c}\text { sCt } \\
\text { "Dependent" }\end{array}$ & $\begin{array}{c}\text { scT } \\
\text { "Disorganized" }\end{array}$ & $\begin{array}{c}\text { sct } \\
\text { "Depressive" }\end{array}$ \\
\hline \multirow{29}{*}{ 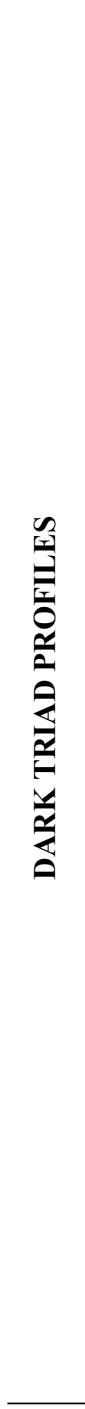 } & mnp "Agreeable" & TYPE & TYPE & ANTI-TYPE & ANTI-TYPE & TYPE & TYPE & ANTI-TYPE & ANTI-TYPE \\
\hline & Observed frequency & 51 & 53 & 5 & 10 & 33 & 42 & 6 & 18 \\
\hline & Expected frequency & 32.6 & 31.5 & 16 & 20.6 & 22.5 & 21.4 & 30 & 43.5 \\
\hline & Chi-square & $10.41 * * *$ & $14.70^{* * *}$ & $7.53 * * *$ & $5.42 * * *$ & $4.88^{* * *}$ & $19.75^{* * *}$ & $19.16^{* * *}$ & $14.96 * * *$ \\
\hline & mnP "Psychopathic" & - & - & - & ANTI-TYPE & - & - & - & - \\
\hline & Observed frequency & 8 & 11 & 7 & 2 & 9 & 7 & 11 & 21 \\
\hline & Expected frequency & 11.4 & 11 & 5.6 & 7.2 & 7.9 & 7.5 & 10.4 & 15.2 \\
\hline & Chi-square & 0.99 & 0 & 0.37 & $3.72 *$ & 0.17 & 0.03 & 0.03 & 2.24 \\
\hline & mNp "Narcissistic" & TYPE & TYPE & - & - & - & - & ANTI-TYPE & ANTI-TYPE \\
\hline & Observed frequency & 32 & 23 & 2 & 9 & 10 & 9 & 3 & 5 \\
\hline & Expected frequency & 13.9 & 13.4 & 6.8 & 8.8 & 9.6 & 9.1 & 12.8 & 18.6 \\
\hline & Chi-square & $23.57 * * *$ & $6.81^{* * *}$ & 3.4 & 0.01 & 0.02 & 0 & $7.48 * * *$ & $9.91 * * *$ \\
\hline & mNP "Psychopathic narcissistic" & - & - & - & - & - & ANTI-TYPE & - & ANTI-TYPE \\
\hline & Observed frequency & 17 & 15 & 10 & 7 & 14 & 3 & 10 & 10 \\
\hline & Expected frequency & 12.9 & 12.4 & 6.3 & 8.1 & 8.9 & 8.5 & 11.8 & 17.2 \\
\hline & Chi-square & 1.34 & 0.54 & 2.18 & 0.15 & 2.95 & $3.52 *$ & 0.28 & $2.99 *$ \\
\hline & Mnp "Machiavellian" & - & - & - & - & - & - & - & - \\
\hline & Observed frequency & 9 & 14 & 7 & 8 & 14 & 14 & 9 & 17 \\
\hline & Expected frequency & 13.7 & 13.3 & 6.7 & 8.7 & 9.5 & 9 & 12.6 & 18.4 \\
\hline & Chi-square & 1.64 & 0.04 & 0.01 & 0.05 & 2.13 & 2.72 & 1.05 & 0.1 \\
\hline & MnP "Anti-social" & ANTI-TYPE & ANTI-TYPE & - & - & ANTI-TYPE & - & TYPE & TYPE \\
\hline & Observed frequency & 5 & 7 & 8 & 15 & 6 & 9 & 28 & 56 \\
\hline & Expected frequency & 20 & 19.4 & 9.8 & 12.6 & 13.8 & 13.2 & 18.4 & 26.7 \\
\hline & Chi-square & $11.27 * * *$ & $7.89 * * *$ & 0.33 & 0.44 & $4.44^{*}$ & 1.32 & $4.99 *$ & $32.00 * * *$ \\
\hline & MNp "Manipulative narcissistic" & TYPE & - & - & - & - & - & - & - \\
\hline & Observed frequency & 16 & 11 & 3 & 9 & 6 & 5 & 8 & 8 \\
\hline & Expected frequency & 9.9 & 9.5 & 4.8 & 6.2 & 6.8 & 6.5 & 9.1 & 13.2 \\
\hline & Chi-square & $3.82 *$ & 0.23 & 0.69 & 1.24 & 0.1 & 0.34 & 0.13 & 2.03 \\
\hline & MNP "Maleficent" & ANTI-TYPE & ANTI-TYPE & TYPE & TYPE & ANTI-TYPE & ANTI-TYPE & TYPE & TYPE \\
\hline
\end{tabular}




$\begin{array}{lcccccccc}- & 11 & 10 & 31 & 34 & 11 & 9 & 62 \\ \text { Observed frequency } & 34.7 & 33.5 & 17 & 21.9 & 24 & 22.8 & 31.9 & 46.3 \\ \text { Expected frequency } & 16.16^{* * *} & 16.49 * * * & 11.56^{* * *} & 6.72^{* * *} & 7.02^{* * *} & 8.36^{* * *} & 28.46^{* * *} & 6.76^{* * *} \\ \text { Chi-square } & \end{array}$

2 Note: $* * * p<.001, * p<.05$.

3 TYPE: the observed cell frequency is significantly greater than the expected (grey fields).

4 ANTI-TYPE: the observed cell frequency is significantly smaller than the expected (black fields).

5 -: the observed cell frequency is as expected (white fields). 


\section{1}

The Dark Cube as an analogy to Cloninger's character cube, showing all eight possible combinations of high/low scores in Machiavellianism, narcissism, and psychopathy.

Note: adapted with permission from C. R. Cloninger. The directions of the arrows represent higher values. $\mathrm{M}$ = high Machiavellianism; $\mathrm{m}=$ low Machiavellianism; $\mathrm{N}$ = high narcissism; $\mathrm{n}$ = low narcissism; $\mathrm{P}=$ high psychopathy; $\mathrm{P}=$ low psychopathy.

\section{The Dark Cube}

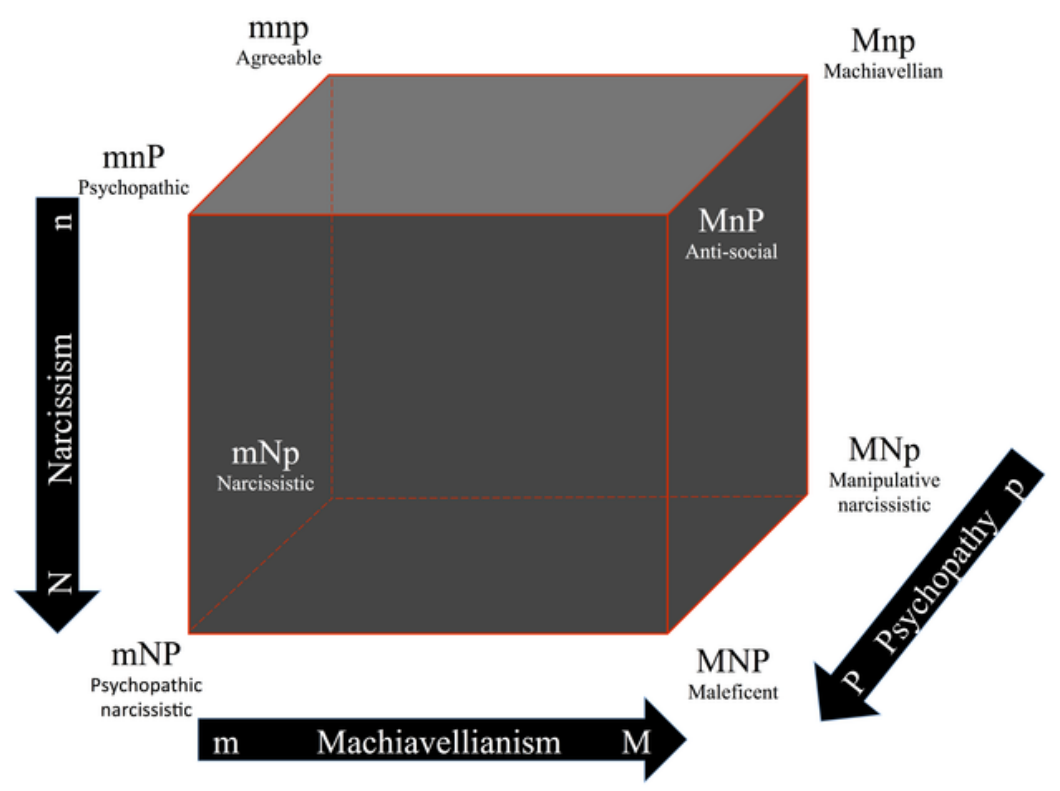


2

Distribution of the different dark character profiles in the Dark Cube.

Note: adapted with permission from C. R. Cloninger. The directions of the arrows represent higher values. $M=$ high Machiavellianism; $m=$ low Machiavellianism; $N=$ high narcissism; $n$ = low narcissism; $\mathrm{P}=$ high psychopathy; $\mathrm{p}=$ low psychopathy. Observe that the scale for neuroticism does not match that of Figure 1 due to technical issues.

*Note: Auto Gamma Correction was used for the image. This only affects the reviewing manuscript. See original source image if needed for review.

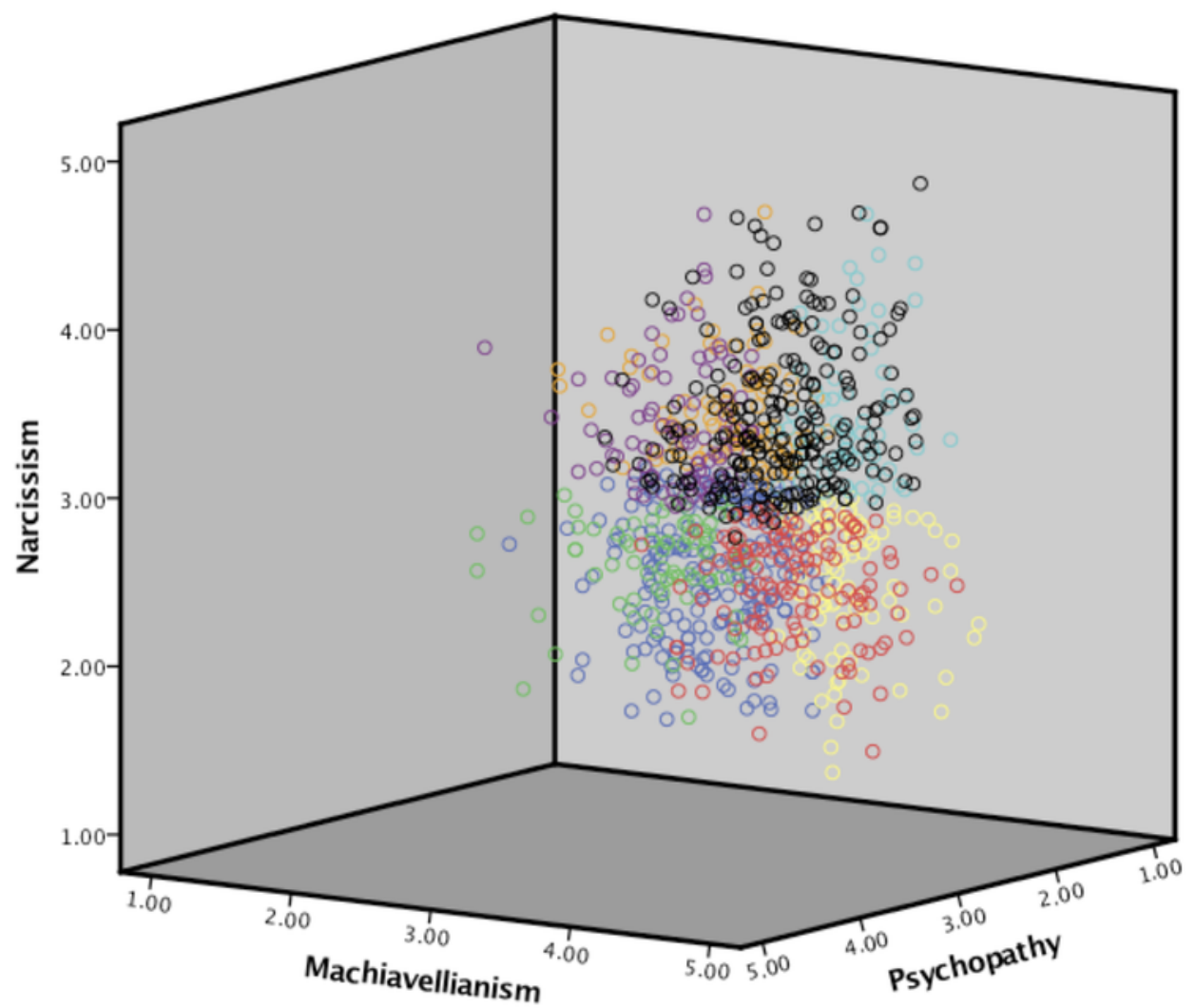

Dark Profile

Omnp Agreeable mnP Psychopathic mNp Narcissistic

OmNP Psychpatic narcissistic Mnp Machiavellian MnP Anti-social

MNp Manipulative narcissistic OMNP Maleficent 